The commercial alloys today are: (1) $\mathrm{Zn}_{18} \mathrm{Al}_{22}$ (optimum $T \sim 250^{\circ} \mathrm{C}$ ), German F.R.

(2) $\mathrm{Al}_{93.5} \mathrm{Cu}_{6} \mathrm{Zr}_{0.5 .}$ (optimum $T \sim 460^{\circ} \mathrm{C}$ ), Britain;

(3) $\mathrm{Al}_{90} \mathrm{Ca}_{5} \mathrm{Zn}_{5}$ (optimum $T \sim 475^{\circ} \mathrm{C}$ ), Canada;

(4) $\mathrm{Ti}_{90} \mathrm{Al}_{6} \mathrm{~V}_{4}$ (optimum $T \sim 950^{\circ} \mathrm{C}$ ), USA.

The $\mathrm{Zn}$-Al monotectic alloy is an example of a duplex-phase microstructure, whereas the Al-Cu-Zr alloy is a good instance of an alloy specifically designed for stabilisation by phase dispersion. A number of duplex steels have been shown to be superplastic, but these have not yet found a use in industrial applications.

Anything which hinders dislocation creep without disturbing diffusional flow (e.g. dissolving tungsten in nickel to lower the stacking-fault energy and thereby hinder dislocation climb, needed if creep is to continue) will enhance superplastic formability. Development of an industrially usable family of superplastic steels and superalloys would be a very desirable prize, as also would, more generally, the perfection of a stably super-finegrained family of light alloys $(d \sim 0.5 \mu \mathrm{m})$ which might offer practical superplastic strain rates approaching $1 / \mathrm{s}$. For this last objective, rapid solidification processing (splat-quenching, see also Cotterill, page 8 ), may prove to be the key.

The above account has not touched at all upon a second form of superplasticity, which is linked with the building up of a system of microstructural internal stresses by thermally cycling an alloy during transformation, either through a phase transition or else by exploiting highly anisotropic thermal expansion in individual grains, as in $\alpha$-uranium. This phenomenon has had major implications for nuclear power (less, now that metallic uranium is being phased out of use). Attempts have been made, unsuccessful up to now, to adapt this form of superplasticity for industrial use.

\section{BIBLIOGRAPHY}

1. Edington J.W., Melton K.N. and Cutler C.P. "Superplasticity", Prog. Mat. Sci. 21 (1976) 61-170.

2. Sherby O.D., Caligiuri R.A., Kayali E.S. and White R.A., "Fundamentals of Superplasticity" in Advances in Metal Processing, eds. J.J. Burke, et al., (Plenum Press) 1981; pp 133-172. 3. "Symposium on the Mechanical, Microstructural and Fracture Processes in Superplasticity", Metallurgical Trans. 13A (1982) 689-744.

4. Padmanabhan K.A. and Davies G.J., Superplasticity (Springer) 1980.

5. Ghosh A.K., "Tensile Instability and Necking in Materials with Strain Hardening and StrainRate Hardening", Acta Metallurgica 25 (1977) 1413-1424.

6. Ashby M.F. and Verrall R.A., "Diffusion-accommodated Flow and Superplasticity", Acta Metallurgica 21 (1973) 149-163.

7. Cheese R. and Cantor B., "Superplasticity in Splat-Quenched Pb-Sn Eutectic", Mat. Sci. and Eng. 45 (1980) 83-93.

\title{
Metallurgy and Superconductivity
}

\author{
J. Muller and J. L. Jorda, Geneva \\ University of Geneva
}

Superconductivity is undoubtedly one of the most remarkable phenomena in solid state physics: an intriguing curiosity at the time of Kamerlingh Onnes' discovery, the superconducting phase transition gradually acquired fundamental importance as the signature of a possible ground state of condensed matter. According to present knowledge, the occurrence of superconductivity in electronically conducting systems is rather the rule than the exception, and the number of superconducting elements, alloys and compounds far outweighs that of, e.g., magnetically ordered substances.

The physics of superconductivity concentrates on understanding the subtle electron interactions responsible for the phenomenon, the pairing in momentum space. However, this aspect is only part of the story. The remaining, no less important part, is connected with applications and the prospecting of yet unknown alloy systems - physical metallurgy.

Superconducting materials, especially those considered for the production of high magnetic fields, are characterized by their critical surface in a three dimensional system defined by temperature, external magnetic field and transport current density. The critical temperature and critical fields are, for the most part, properties of the composition within a homogeneous phase of the material (the latter may be an equilibrium phase or a metastable one); the microstructure has not much influence because the superconducting coherence length is usually much smaller than dimensions such as grain size. On the other hand, the critical current density $J(H, T)$ depends - even crucially - on microstructural features such as grain size, dislocation networks, precipitates etc. There are thus at least two kinds of "metallurgy" which come into play, the first dealing with problems of phase stability and the second focussing on defect structures.

Phase Diagrams \& Superconductivity

Many superconductors - particularly the most interesting, those with a high critical temperature $T$ - show important variations of their properties with the method of preparation and with heat treatment, and the first step in understanding or predicting such variations is to examine the details of their alloy phase diagrams. The niobium-gallium system which exhibits an A 15-type structure when the composition is near $\mathrm{Nb}_{3} \mathrm{Ga}$, illustrates such behaviour. If one measures $T_{c}$ for one and the same specimen as a function of the annealing temperature $T_{A}$, a complicated curve is obtained as shown in Fig. 1. $T$ varies between the extremes of $9 \mathrm{~K}$ and $21 \mathrm{~K}$ and, moreover, will not exceed about $15 \mathrm{~K}$ unless rapidly quenched after the annealing treatment.

To attain the maximum value, the precise homogeneous phase composition is first obtained by appropriate annealing and quenching, and this is followed by a low temperature annealing (branch $\mathrm{I} b$ in Fig. $1)$, performed in a region where segregation is avoided, so ensuring an improvement in the long range order parameter.

The main reason why $T_{c}$ is so dependent upon the detailed heat treatment processes is that the peritectically formed A 15 compound has a strongly temperature-dependent phase limit at the $\mathrm{Ga}$ rich side as can be seen in Fig. 2. The stoichiometric concentration of $25 \% \mathrm{Ga}$, a prerequisite for a high $T_{c^{\prime}}$ is confined to a narrow spike near the eutectic temperature of $1740^{\circ} \mathrm{C}$.

The more intriguing niobium-germanium system is governed by a topologically similar phase diagram around the superconducting A 15 phase. However, the limit falls short of the composition $\mathrm{Nb}_{3} \mathrm{Ge}$ by 2 atomic percent even at the eutectic temperature. Moreover, the tendency towards segregation below $T_{E}$ is so strong that the strategy "optimum quench plus ordering anneal" does not work at all here. Yet suitably prepared, $\mathrm{Nb}_{3} \mathrm{Ge}$ holds the record for $T_{c}$. It is then clear that the constraints of three-dimensional equilibrium thermodynamics have to be overcome right from the beginning of material synthesis. This can be done by growing films by sputtering or physical or chemical vapour deposition. The success of these techniques relies

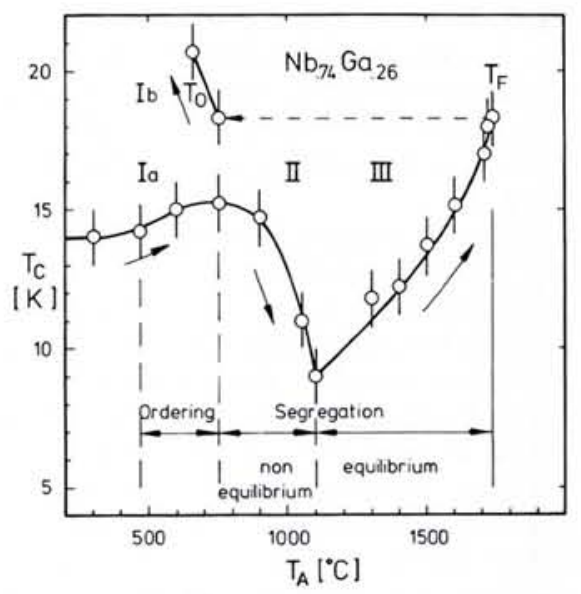

Fig. 1 - The superconducting critical temperature $T_{c}$ of the niobium-gallium $A 15$ phase vs. the annealing temperature $T_{A}$. 


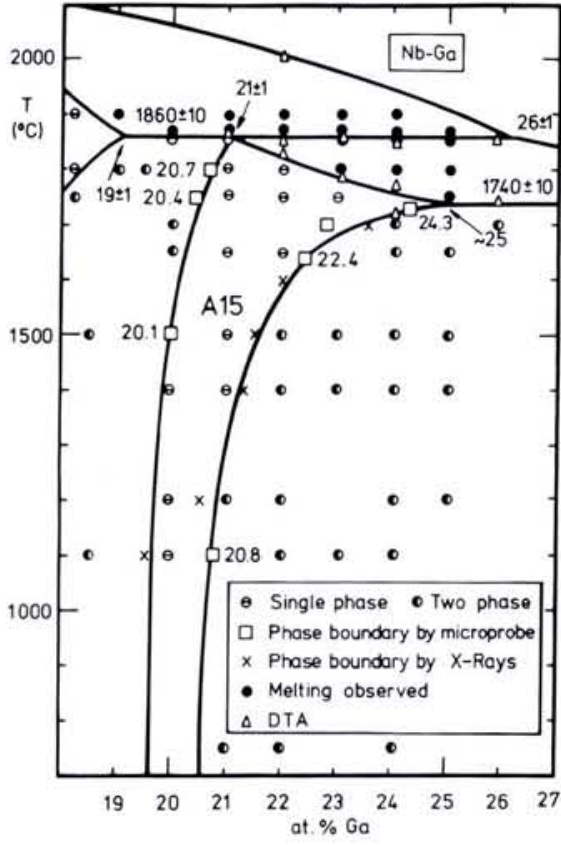

Fig. 2 - A detail of the Nb-Ga phase diagram the explanation of Fig. 1.

essentially on surface processes since the substrate is held at moderate temperatures. Indeed, the surface mobility of the impinging atoms is considerable - allowing the formation of a well ordered structure while bulk diffusion is virtually cut off. Of course, this does not explain the sizable shift of the homogeneous phase boundaries but one may infer, nevertheless, that surface energy plays a determining role in the competition between different crystal structures. A kind of pseudo-epitaxial mechanism also appears to be at work.

One important lesson the metallurgists have learned from their study of superconducting materials is that alloy phase diagrams should not be terminated at or above ambient temperature. Structural phase transitions occur at surprisingly low temperatures, although of course they must be of a diffusionless type. A now classical example is the Batterman-Barrett transformation observed in $\mathrm{V}_{3} \mathrm{Si}$ and $\mathrm{Nb}_{3} \mathrm{Sn}$ at $21 \mathrm{~K}$ and 40 - $50 \mathrm{~K}$, respectively. The low temperature modification corresponds to a tiny tetragonal deformation of the "high temperature" cubic structure. Many physical properties are markedly affected by this distortion but, interestingly, the superconducting properties undergo only minor changes.

A whole class of similar transformations has been found more recently, notably in Laves-type and Chevrel-type phases, and in the latter, the consequences appear to be quite dramatic. The rhombohedral $\mathrm{PbMo}_{6} \mathrm{~S}_{8}$ is a high $T_{c^{\prime}}$, high critical field superconductor, whereas if one replaces the divalent lead ion by alkaline earths or divalent europium, the resultant compounds become semimetals at low temperatures and do not superconduct. Closer inspection has revealed the reason for this: a very small rhombohedral to triclinic distortion appears well below room temperature, completely modifying the electronic structure of the crystal - a kind of superBatterman-Barrett effect. Marginal phase stability may be a feature of a number of narrow band systems and therefore of many potential new superconductors.

\section{Influence of Microstructure on Super- conducting Properties}

Ever since superconducting wires or tapes have been wound into coils, the successful generation of high fields has depended on a careful control of the defect structure. This is necessary because resistanceless current flow in type II superconductors (those characterized by a partial magnetic field penetration) depends on effectively pinning down the flux line vortices. Such pinning problems were encountered long ago in other contexts, for example in studies of mechanically strong or hard ferromagnetic materials.

In superconductors, one has to take into account the efficiency of individual pinning sites and their (inhomogeneous) distribution. However, evaluating the volume pinning force, and therefore the critical current densities, presents a complicated "summation problem" and still awaits a complete solution. The practical ductile superconductors such as $\mathrm{Nb}$-Ti achieve a sufficiently high value of $J_{c}$ through having a fine dislocation cell structure, formed by a sequence of appropriate cold work and heat treatment. The production techniques for this material are now well developed, although absolute control over kilometers of wire required for large-scale applications is still a non-trivial metallurgical problem.

A 15-type and other compounds offer higher critical fields than $\mathrm{Nb}$ - Ti but in these brittle substances, dislocations are almost absent. Fortunately, the grain boundaries also ensure efficient pinning, presumably through an increased electron scattering frequency in the interface regions. High current densities are consequently still possible if the grain size is small enough typically of the order of $0.1 \mu \mathrm{m}$. Inspection of the $J_{c}$ values shown in Fig. 3 will confirm that materials like $\mathrm{Nb}_{3} \mathrm{Sn}$ are indeed satisfactory in fields of say $15 \mathrm{~T}$, but there are many other problems connected with the design of field-generating configurations. Strain sensitivity, mechanical and thermal properties of the composites, etc., are subjects which again link the physics of superconductors with practical metallurgy.

What of the future for high field superconductors? Interestingly enough, materials with critical fields about twice as high as those of the A 15's (see the $x$-axis of Fig. 3) are already at hand. Some of the Chevrel-type compounds, typically represented by $\mathrm{PbMo}_{6} \mathrm{~S}_{8}$ already mentioned above, owe their exceptional upper critical field to a low Fermi velocity, a high $T$ and a high resistivity at low temperatures. The problem remains to boost up current densities significantly above the level presently achieved. No fundamental reason can be invoked why this should not be possible but a concentrated metallurgical research effort is clearly needed.

\section{Universiteit van Amsterdam}

\section{The University of Amsterdam has an opening for the position of professor of experimental physics $(\mathrm{m} / \mathrm{f})$}

The appointed candidate's research will constitute part of the research program of the "Natuurkundig Laboratorium" which concerns condensed matter physics. He/she will be expected to take initiatives in this field which can be realized within the broad possibilities offered by the laboratory. Facilities are available for experiments at low temperatures, high magnetic fields, and high pressures; for the measurement of optical, magnetic, and thermodynamic properties; for the preparation of samples, etc. Present areas of research include the properties of solid hydrogens, magnetism and electronic structure of intermetallic compounds, defects in semiconductors and in intermetallic compounds.

The successful candidate should have didactic skills enabling him/her to participate in the teaching activities of the Department of Physics and Astronomy. Involvement in Departmental administration will also be expected.

Salary min. Dfl. 6.561.- max. Dfl. 9.283,-- per month (Dutch Civil Servants Code).

Applications including a curriculum vitae, a list of publications and the names and addresses of a few referees, willing to provide information concerning personal and scientific qualifications should be addressed before March 11983 to the chairman of the Nominating Committee.

Prof. Dr. P.F. de Châtel, Natuurkundig Laboratorium, Valckenierstraat 65 . 1018 XE Amsterdam, the Netherlands, quoting number 6333. Tel. 020 - $5222797 / 2716$.

Those wishing to draw attention to potential candidates are also welcome to contact the Committee Chairman not later than March I 1983 


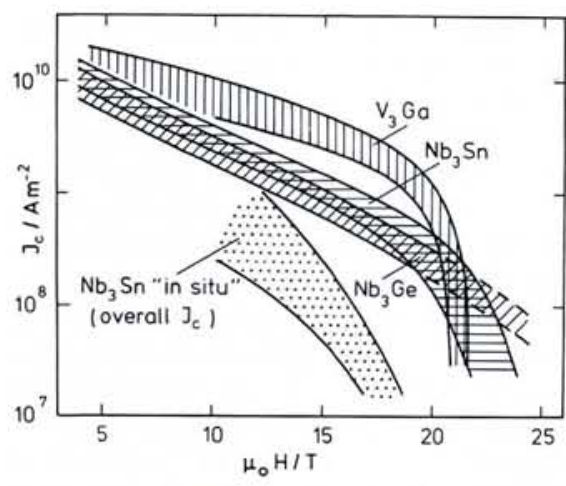

Fig. 3 - Typical short sample critical current densities vs. transverse magnetic field at $4.2 \mathrm{~K}$

Superconductivity: Tool in Metallurgy

The work with superconducting alloys is not just a race towards better conductors. When investigating new alloy systems, metallurgists make use of a number of physical techniques, and it is precisely because superconductivity is so wide-spread in metallic materials that its observation is so useful in ascribing the correct compositiontemperature coordinates of certain phase fields. We thus have an additional analytical tool of extremely good sensitivity.

The interesting point is that the sample quantity needed to detect a well defined diamagnetic transition is incredibly small. The absolute record in this respect belongs to the SQUID magnetometer - a device kindly provided by superconducting flux quantization. With this, it is almost always possible to pick out from a polycrystalline specimen, for isolated investigation, a barely visible single crystal. Another attractive possibility of observing the second order transition at $T_{c}$ - even in poor quality samples - is the measurement of the specific heat. In this case, the analysis of the anomalous electronic part yields information on possible inhomogeneous phase distributions or concentration fluctuations. Fortunately, again, modern low temperature calorimetric techniques allow the use of very small specimens, in favourable cases, a mass of the order of $1 \mathrm{mg}$ is enough. Of course, in many routine investigations, such elaborate experimental methods are not necessary, and quite often, a simple permeability-temperature scan on powdered specimens may suffice. It is instructive to note that in the past, when measurements made on superconducting transitions as a function of alloy composition appeared to be inconsistent with some well established rules, the explanation was always simple: the previously constructed phase diagram was wrong.

Superconducting technology, because of its unique possibilities, is expected to grow steadily in importance. A great deal of metallurgy will be needed to cope with major problems. From a somewhat different view-point, superconductivity still assumes another function: that of a motor in materials science.

\title{
Metallic Glasses
}

\author{
R.M.J. Cotterill, Lyngby \\ The Technical University of Denmark
}

Covalently-bonded glasses, such as the common silicate varieties, have been in use for at least 4000 years. Glass beads, dating from about $2500 \mathrm{BC}$, have been found in Egypt and other parts of the Near East, although exploitation of the plasticity of hot glass is more recent. These materials have so dominated the scene that the existence of the glassy state was believed to be intimately connected with the presence of covalent bonds. Glass has long been known to be a supercooled liquid, and covalent bonding was regarded as a pre-requisite if crystallization was to be avoided. This attitude has recently undergone radical revision. With the production of metallic examples, it has suddenly transpired that a glass need not be transparent, brittle or insulating. Glass possesses a unique lack of order, and it is this atomic-level attribute which is now acknowledged as the defining characteristic of this type of material.

It is not often that branches of science and technology as venerable as metallurgy and glass fabrication witness a genuine revolution. When both these fields simultaneously experienced such an upheaval, in 1960 , it was not surprising that the repercussions were widespread. In producing the first appreciable amounts of metallic glass, Pol Duwez, William Klement, and Ronald Willens effectively rewrote the definitions of both classes of material. Until their breakthrough, the distinction between these forms of matter had been reflected in a marked difference in the chemical compositions. The workhorses of metallurgical practice are iron, copper, zinc, aluminum, lead, nickel, and cobalt. The familiar glasses, on the other hand, are formed from compounds, in which silicon, oxygen, and sodium are particularly prominent.

The free energy barrier separating the non-crystalline and crystalline states of metals is rather small, and slow cooling of a metallic melt does not yield a glass. It has long been known however, that metals are capable of exhibiting properties other than those of their natural state. The simple trick is to cool them so rapidly that a nonequilibrium state is frozen in, this process being known as quenching. A cooling rate of tens of millions of degrees per second is required to trap a metal in its amorphous state, and this can be achieved in a variety of ways. The original method involved propelling molten globules against a cold flat surface, a process known as splat quenching. More recent methods include pouring the liquid between rapidly counterrotating drums and squirting a thin jet of the liquid against the cooled surface of a revolving cylinder. The last of these produces a continuous ribbon, at rates of up to $2000 \mathrm{~m} / \mathrm{min}$ and probably holds the greatest promise for commercial exploitation. The quite different technique of vapour condensation is also effective, and there were reports in the 1930s, notably by J. Brill and J. Kramer, of metal glass formation by this method. Credit for the revolution must also be accorded to these pioneers.

Congealed metallic melts are frequently referred to as amorphous alloys, emphasizing the fact that they are mixtures rather than pure metals. They have invariably belonged to one of two groups: a transition, or a noble metal with a smaller metalloid, or a mixture of two transition metals. Examples of the first type are $\mathrm{Au}_{75} \mathrm{Si}_{25}$ (the first combination studied by Duwez, Klement, and Willens), $\mathrm{Pd}_{80} \mathrm{Si}_{20}$ and $\mathrm{Fe}_{80} \mathrm{~B}_{20}$. The other group includes $\mathrm{Ni}_{60} \mathrm{Nb}_{40}$ and $\mathrm{Cu}_{66} \mathrm{Zr}_{34}$. Many of the more recently developed metallic glasses have more complicated compositions, such as $\mathrm{Fe}_{75} \mathrm{P}_{16} \mathrm{~B}_{6} \mathrm{Al}_{3}$ and $\mathrm{Pd}_{16} \mathrm{Au}_{66} \mathrm{Si}_{18}$. Production cost is relatively low because the glassy alloys are formed directly from the molten metal, so it is possible to bypass expensive and energy-consuming stages such as casting, rolling, and drawing, which have been necessary when working with the crystalline state of the material. One technological limitation arises from the fact that a metallic glass reverts to the crystalline form if the temperature is raised to roughly half the melting point on the absolute Kelvin scale.

One of the best known models of the liquid state is that usually accredited to John Bernal, although variants of the same approach had been discussed by Peter Debye and $\mathrm{H}$. Menke, and also by Joel Hildebrand and W.E. Morrell, both in the 1930s. Bernal's version, dating from 1959, involved pouring spheres into an irregularly-walled container to produce what is known as random close packing. As John Finney subsequently demonstrated, the model is actually more applicable to metallic glasses, particularly when the structure is produced with two different sizes of sphere, with appropriate diameters. It must be emphasized that such models do not exhibit the total randomness of an ideal gas, because the dense packing imposes quite severe geometrical constraints. A certain degree of short-range order exists, even though longrange order is completely lacking. This has led some to conclude that metallic glasses are actually polycrystals with a grain size of near atomic dimensions. Indeed, it has 